\title{
Rapid Tools Compensation in Sheet Metal Stamping Process
}

\author{
Lorenzo lorio $^{1, a}$, Matteo Strano ${ }^{2}$ and Michele Monno ${ }^{2}$ \\ ${ }^{1}$ MUSP Lab, Piacenza, Italy \\ ${ }^{2}$ Politecnico di Milano, Dipartimento di Meccanica, Milan, Italy
}

\begin{abstract}
The sudden growth of additive manufacturing is generating a renovated interest towards the field of rapid tooling. We propose a geometrical compensation method for rapid tools made by thermoset polyurethane. The method is based on the explicit FEM simulation coupled to a geometrical optimization algorithm for designing the stamping tools. The compensation algorithm is enhanced by considering the deviations between the stamped and designed components. The FEM model validation has been performed by comparing the results of a DOE done at different values of press force.
\end{abstract}

\section{Introduction}

In the conventional deep drawing and stamping processes, a tooling setup made of a die, a punch and a blankholder is traditionally used. Over the years, many different types of flexible sheet forming processes have been developed in the industry, in order to improve the process, and especially in order to compress the tooling production times and costs. Single point and double point incremental forming processes 1 have been invented and are continuously being developed to this purpose. As another cost saving option, metal tools can be replaced with a rubber membrane. In the Guerin process, a movable thick rubber pad is pressed against a die 2 or a punch 3. The Marform variant of the Guerin process, an active blankholder 4 is used. In flexforming with a fluid cell, a rubber diaphragm is pressurized by a fluid or by a bladder 5. In deep drawing flexforming, a movable punch is used, too 6. In multi-point stretch forming 7, stretch forming clamps are used with the sheet bent over a flexible die, made by a raster of metallic movable pins.

In the last few years, the tremendously rapid growth of additive manufacturing is changing completely the way of thinking about and designing functional parts. At the same time, a renovated attention is being given to rapid tooling technologies 8 , which offer cost-efficient and innovative solutions for improving the sheet metal forming processes.

The rapid tooling method proposed in the present work is to machine polymeric boards, made by thermoset polyurethane, which in a few minutes can be machined into a forming tooling setup (punches, dies and blankholder). Very scarce scientific literature is available with this respect, to the authors' knowledge. One of the previous research works concerning all-polymeric forming tools with experimental and numerical analysis are by Park and Coulton of Georgia Tech 9, in the mid of years 2000. Sheet metal forming processes performed only with plastics tools are only used as prototyping methods, i.e. in prototyping job shops or for artistic uses by jewellers and metalsmiths 10 .

The main advantage in using rapid polymeric tools is due to their low required machining energy and cost. The most expensive material used in this paper (commercial name Necuron 1300) has a comparable cost per unit volume, expressed in $€ / \mathrm{m} 3$, to an AISI 1040 steel. However, the material removal rate in machining is more than 4 times faster, with a negligible tool wear in forming applications with thin sheet metals.

The main disadvantage of polymeric tools is that deflect elastically (or plastically, if they are not properly designed) under the forming forces. As a consequence, the final geometry of the formed part is difficult to predict, not only because of the usual springback, but also because of the deflection of the tools. For this reason, a numerical die compensation technique is required, able to suggest the correct shape for the tools, in order to keep the part within tolerances.

The most frequently used methods for die compensation are the displacement adjustment (DA), the surface controlled overbending (SCO) and the Force Descriptor Method (FDM). The displacement adjustment is very effective; the tool nodes are displaced in the opposite direction of the blank springback 11. The deviations calculation is done between the correspondent nodes of the simulated and the designed blank. This means that no remeshing is possible or, alternatively, any new mesh must be remapped with reference to the mesh of the designed part. For this reason, the DA method is

${ }^{a}$ Corresponding author: lorenzo.iorio@musp.it 
frequently used for simple $2 \mathrm{D}$ forming cases 12 , where a small number of nodes must be mapped or remeshing is not even required.

The surface controlled overbending algorithm performs the calculation directly on the tools CAD but, again, the calculation complexity makes the method inaccurate on a complex surface with high degree 13 .

The Force Descriptor Method proposed by Karafillis and Boyce 14 is an iterative method based on the evaluation of the internal forces of the component, but the algorithm suffers from lack of convergence, especially in symmetric cases or limit values of springback.

The authors have proposed, at the ASME-MSEC 2015 conference, a compensation algorithm in combination with an FEM model 15, which allows to predict the deformation of the tools and to determine the required compensation in case the part falls outside the initial design tolerances. The proposed algorithm is inspired by the DA method, but improves the distance calculation by evaluating the normal distance from the tools node to the interpolated blank surfaces. Unlike the standard DA, there is no need to keep track of predetermined couples of nodes. The calculation of the normal distance solves the main disadvantage of the DA algorithm, allowing the applicability of the method also for $3 \mathrm{D}$ complex components. The results presented at the ASME paper were encouraging, but the method was tested with a relatively simple part. In the present work, a different test case, with greater geometrical complexity, has been used and the compensation algorithm has been significantly modified.

In the following Sections we will describe the experimental test case and the FEM model. Then, the development of the compensation algorithm will be described, in terms of mathematical formulation and algorithmic solutions. Finally, the validation and compensation results will be presented.

\section{Description of the experimental test case}

The test case (Figure 1) is a stamped component with a double symmetry plane and some geometrical features which are predictably hard to be obtained in a stamping process with flexible tools. The constant radii (R6 in Figure 1) are very small, i.e. difficult to be obtained in this kind of processes, because the deformable tools usually tend to slightly deform by compression in corner regions. The presence of the central, diamond shaped, depression adds complexity to the overall geometry of the process.

The material chosen for the test case is Al1050, annealed, with $1.5 \mathrm{~mm}$ wall thickness. The reference geometry of the part given in Figure 1, which will be called "designed geometry", is the starting point for determining the geometry of the deformable tools. An initial guess geometry of the deformable tools is built as an offset of the surfaces of the test case part; the iterative compensation algorithm will suggest a modification of this initial geometry. The deformable tooling setup
(Figure 2) is made of a die, a punch (blue tools) and a blankholder (orange tool).
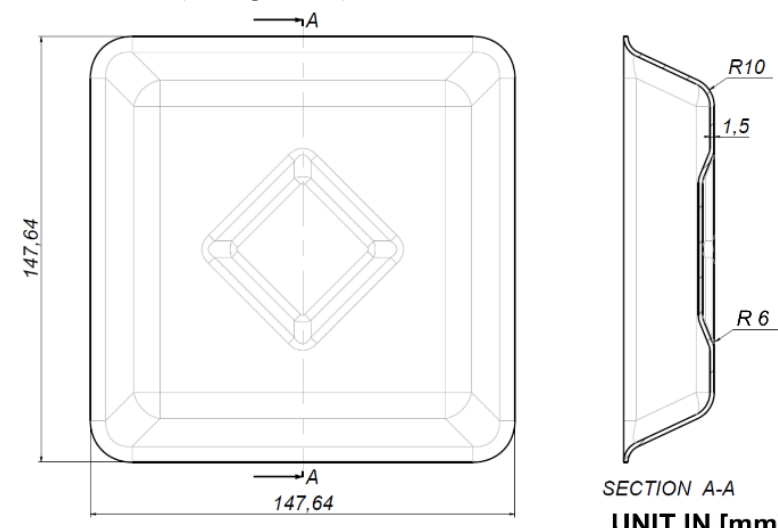

UNIT IN [mm]

Figure 1: geometry of the test case used for developing the optimization algorithm.

The base of punch (orange) has been generated by using the material milled to the centre part of the blank holder, in order to reduce the cost of the required resin.

The polyurethane materials chosen for testing the deformable tools are: Necuron 1300 for the punch basement and blankholder; Miketool 1440 for the die and punch. The material properties are discussed in the following Section. The estimated cost of the polyurethane die is about $€ 990$ (including machining costs). The same die, made by Zamak 2 (which is a low cost alloy typically used for prototyping tools) would cost about $€ 1860$. In Figure 3 a quantitative comparison of the tools price in terms of total manufacturing and material costs has been presented, in order to show the economic advantage related to the use of polyurethane tools.

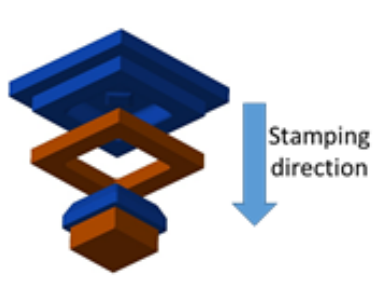

(a)

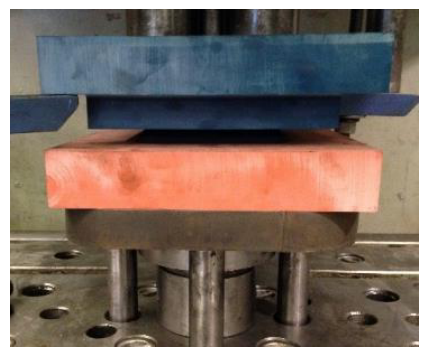

(b)
Figure 2: (a) setup of the three deformable tools (punch, blankholder and die), along with the connecting plates (b).

Figure $2 \mathrm{a}$ shows the direction of the stamping process. The stamping process is made of three steps:

1 Holding: the blank is placed on the blankholder and then the die moves downward for holding it;

2 Stamping: the die pushes the blank against a fixed punch and against the blankholder with a maximum force of $980 \mathrm{kN}$; the maximum available reaction force of the blankholder is $392 \mathrm{kN}$.

3 Springback: the tools are released and both the tools and the blank recover the elastic deformation. 


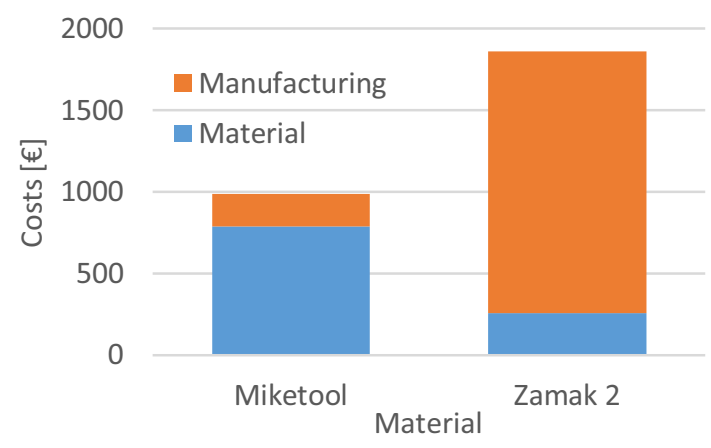

Figure 3: costs comparison between Miketool (polyurethane) and Zamak2 for making the die in the experimental case.

An experimental plan has been designed with variable levels of the blankholder force BHF, without replications (Table 1). The plan is aiming at determining which level of maximum BHF could determine failure of the parts (by either wrinkling or fracture) or failure of the tools (by fracture or plasticization). It is also aimed at determining the influence of BHF on the geometrical accuracy of the obtained parts.

Table 1: experimental plan

\begin{tabular}{|c|c|c|}
\hline $\begin{array}{c}\text { Experiment } \\
\text { no. }\end{array}$ & $\begin{array}{c}\text { Maximum } \\
\text { BHF } \\
{[\mathrm{kN}]}\end{array}$ & $\begin{array}{c}\text { Maximum error } \\
\text { measured on the } \\
\text { A-A cross section }[\mathrm{mm}]\end{array}$ \\
\hline 1 & 4.9 & -0.20 \\
\hline 2 & 9.8 & -0.21 \\
\hline 3 & 14.7 & +0.27 \\
\hline 4 & 19.6 & +0.14 \\
\hline 5 & 23.5 & -0.28 \\
\hline 6 & 24.5 & -0.26 \\
\hline 7 & 25.5 & $\begin{array}{c}\text { Failure by fracture, } \\
\text { part not measured }\end{array}$ \\
\hline
\end{tabular}

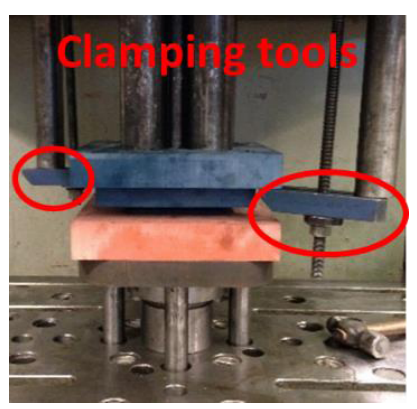

(a)

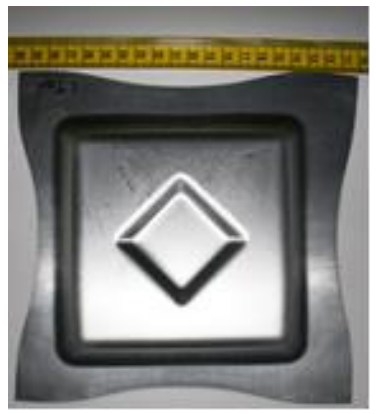

(b)
Figure 4: (a) stamping tools mounted on the press; (b) the stamped component made with the initial geometry of the tools.

The experimental results showed that no macroscopic wrinkling occurred at any level of $\mathrm{BHF}_{\text {max }}$.

The part at experimental condition no. 7 failed by fracture, hence the safe limit to fracture for $\mathrm{BHF}_{\max }$ was assessed at $24.5 \mathrm{kN}$. All stamped parts have been measured with a CMM. All measured parts present some deviations (errors) from the designed part. The profiles measured at the symmetry plane (cross section A-A in
Figure 1) are shown in Figure 5. Not all profiles are shown, in order to improve the clarity of the figure, not making too crowded with lines. The error profiles are not perfectly symmetrical around the centre of the chart because the clamping conditions of the blankholder were not perfectly symmetrical (Fig. 4a), hence different drawin values have been allowed in different directions. Experiment number 6 (which has the largest admissible BHF value) presents the smallest possible error in the central region, but a relatively large error on the corners. The low error in the central part is due to increased stretching, while the larger error on the corners is due to compression of the plastic die corners. Experiment no. 4 seems to be the best compromise but it is not better than other profiles along the whole cross section. Indeed, this is the most interesting remark coming out the of the comparison of the error profiles: there is not a single BHF level able to generate an error which is consistently smaller throughout the whole cross section. The errors measured in some other regions of the part, away from the A-A cross section are even larger than the ones shown in Fig. 5. In order to reduce the amount of geometrical errors, a tool shape geometry compensation procedure is required, in combination with an FEM model of the process.

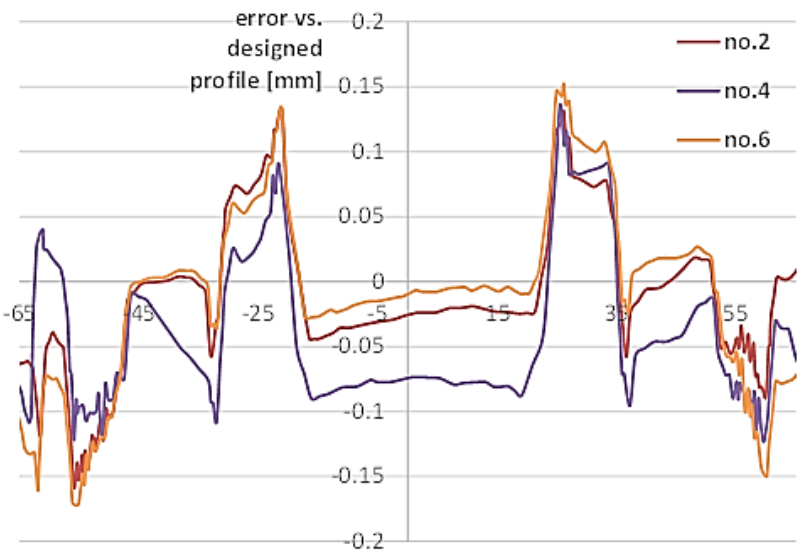

horizontal coordinate along symmetry axis $[\mathrm{mm}]$

Figure 5: error on the nominal profile along cross section A-A

\section{FEM model description}

The FEM model has been implemented with the commercial code PAM-STAMP 2G V2015.1. In order to evaluate the deformation of the polyurethane tools, their mesh is made of four-node tetrahedral solid elements. The solid mesh of the plastic tools is created with VisualMesh 11 by using an automatic meshing module. The surface curvature criterion has been used to determine the element density (finer mesh is generated in areas of higher surface curvature). The number of elements and the number of nodes for each tool is shown in Table 2. A good discretization is important for the accuracy of the FEM simulation, but it is also very important for the application of the compensation algorithm described in the following Section. 
The edge length of the squared blank is $200 \mathrm{~mm}$ and the surface area of each initial element is $400 \mathrm{~mm}^{2}$ with a total number of 100 initial elements. Every refinement step splits 1 quadrangular element into 4 smaller quadrangular shells, by an automatic refinement algorithm. Each element can be split up to 5 times.

Table 2: number of elements and nodes used for meshing the tools.

\begin{tabular}{|c|c|c|}
\hline Tool & N. elements & N. nodes \\
\hline Die & 304291 & 58952 \\
\hline Blankholder & 19898 & 4797 \\
\hline Punch & 387548 & 71129 \\
\hline Punch Base & 12064 & 2641 \\
\hline
\end{tabular}

Table 3: material properties of the polyurethane tools and aluminum blank.

\begin{tabular}{|c|c|c|c|c|c|}
\hline Material & $\begin{array}{c}\text { Young } \\
\text { Modulus } \\
M P a\end{array}$ & $\begin{array}{c}\text { Linear } \\
\text { elastic } \\
\text { limit } \\
M P a\end{array}$ & $\begin{array}{c}\text { Yield } \\
\text { Stress } \\
M P a\end{array}$ & $\begin{array}{c}\text { Poisson } \\
\text { coeff. }\end{array}$ & $\begin{array}{c}\text { Density } \\
\mathrm{g} / \mathrm{cm}^{3}\end{array}$ \\
\hline Al1050 & 72000 & & 33 & 0.335 & 2.73 \\
\hline $\begin{array}{c}\text { Necuron } \\
1300\end{array}$ & 2317 & 35 & 68 & 0.340 & 1.15 \\
\hline $\begin{array}{c}\text { Miketool } \\
1440\end{array}$ & 2950 & 57 & 81 & 0.340 & 1.20 \\
\hline
\end{tabular}

The tensile test data of the blank have been retrieved in the "CES EduPack" material database, since the Al 1050 is a very common material. Tensile data of the tool materials can be found in the literature too 16. Extensive quasi-static tensile and compressive tests have been performed on the polymeric tools in order to determine their elastic modulus. The resulting values for the elastic modulus and the Poisson coefficients are reported in

Table 3. The blank material is modelled as elasticplastic. The coefficients of the Krupkowsky's strain hardening law used in the simulations:

$$
\sigma=K \cdot\left(\varepsilon_{0}+\varepsilon_{\mathrm{p}}\right)^{\mathrm{n}}
$$

are $\mathrm{K}=126.4 \mathrm{MPa}, \mathrm{n}=0.193, \varepsilon_{0}=0.00093$.

The simulation starts at the end of the holding stage in which the blank is held between the die and the blankholder (Figure 6a).

The contact algorithm between the objects is automatically defined by the software taking into account a Coulomb friction coefficient of 0.29 , in order to better simulate the interaction conditions between polyurethane and aluminium materials.

As shown in Figure 4a, the plastic tools are enclosed within metal clamping tools which connect and lock the tools to the press machine. The position of the locking tools along the diagonal of the die does not allow the application of the symmetry to the FEM model. In order to correctly simulate the effect of each clamp on the deformable tools shown in Figure 4a, proper boundary conditions have been applied only on specific nodes and surfaces.

For the die, an imposed displacement with constant velocity is applied on the upper and on some nodes corresponding to the clamping tools (Figure 4a). The
BHF is applied on the lower surface, in order to simulate a uniform distribution of the pressure; finally, a fixed displacement is applied on the lower nodes of the punch base (Figure 6b). The nodes between the punch and the base have been fused in order to simulate the glued contact between the upper surface of the base (orange) and the lower surface of the punch (pink). The springback simulation is run by the FEM software with the "Advanced Implicit" algorithm, by locking three nodes on the stamped component.

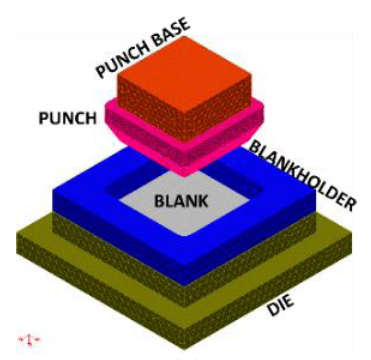

(a)

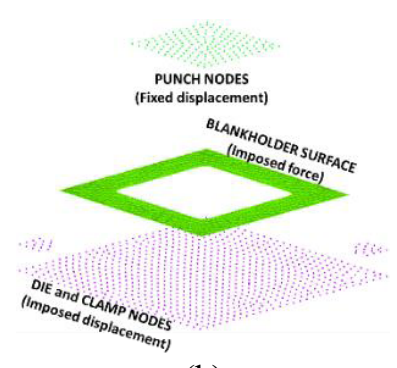

(b)
Figure 6: scheme of the simulation setup (a) and boundary conditions (b) applied to the FEM simulation.

\section{Compensation algorithm}

The main response variables in a stamping process with deformable tools are the deviations between the geometry of the real stamped component and the designed one. The proposed algorithm suggests the compensation to be applied on the plastic tools, in order to obtain the minimization of the deviations. The compensation algorithm iteratively runs the following two main steps:

STEP I. FEM simulation: the user runs a stamping simulation with springback, and a mesh refinement stage. The blank mesh is built with shell elements which represent the middle surface of the part, i.e. half of the thickness must be added in the normal direction in order to model the actual outer or inner surface of the part. As a first guess, in this initial run, the deformable tools are built by offsetting the designed part by half of the initial sheet thickness. During refinement, the meshes of the simulated component and the designed one are both regenerated with shell elements of approximately uniform side length. The final mesh refinement stage is very useful for performing simpler and more accurate computations at the next step II.

STEP II. Compensation: the proposed automatic algorithm has been coded within $\mathrm{C}++$. The routine takes the following inputs: the refined triangular shell mesh of the simulated and the designed parts, the outer contour of the simulated part, the external surface shell mesh of the tools in ascii. The tool geometry is modified according to the following 4 sub-steps:

II.1 Blanks mesh import and surface fitting: the meshes of simulated and designed components are imported. The nodal cartesian coordinates of both 
meshes are converted into parametric coordinates, with the library implemented in 17 , as:

$$
\Sigma_{s}=\left\{\begin{array}{l}
x_{s}=x_{s}(u, v) \\
y_{s}=y_{s}(u, v) \\
z_{s}=z_{s}(u, v)
\end{array} \quad \Sigma_{d}=\left\{\begin{array}{l}
x_{d}=x_{d}(u, v) \\
y_{d}=y_{d}(u, v) \\
z_{d}=z_{d}(u, v)
\end{array}\right.\right.
$$

The parametric surfaces are reconstructed with the Multilevel B-spline Approximation (MBA) 18, where an algorithm models the surface with a recursive refinement of the B-spline knots depending on the level of desired accuracy (k). This fitting allows handling the designed and simulated objects not as numerical meshes with their nodes, but as mathematical surfaces.

II.2 Tools and blank contour import: the external surface nodes of the tools and the outer contour of the simulated component are imported. The contour is useful to define the domain of the compensation.

II.3 Compensation. This step II.3 is repeated for both tools: the punch and the die. Figure 7 shows a 2D graphical representation of the computed quantities. The distances $\delta_{\mathrm{d}}$ and $\delta_{\mathrm{s}}$ between the generic tool (punch or die) node $\vec{T}_{0}\left(x_{t 0}, y_{t 0}, z_{t 0}\right)$, and the two fitted surfaces (simulated and designed) are computed through the conjugate gradient minimization algorithm 17. These distances are 3D vectors, with components in the cartesian coordinate system used for the simulation setup:

$$
\begin{aligned}
\vec{\delta}_{s} & =\left[x_{s}, y_{s}, z_{s}\right] \\
\vec{\delta}_{d} & =\left[x_{d}, y_{d}, z_{d}\right]
\end{aligned}
$$

The deviation vector between the simulated and designed surfaces is defined as

$$
\vec{\delta}_{c}=\left(\vec{\delta}_{d}-\vec{\delta}_{s}\right)
$$

With the components:

$$
\vec{\delta}_{c}=\left[x_{\delta c}, y_{\delta c}, z_{\delta c}\right]
$$

The tool nodes are moved from the original position $\vec{T}_{0}$ by the quantity $\vec{\delta}_{c}$. The final position of the compensated tool node is defined as:

$$
\vec{T}_{c}\left\{\begin{array}{l}
x_{T c}=x_{T 0}+\lambda x_{\delta c} \\
y_{T c}=y_{T 0}+\lambda y_{\delta c} \\
z_{T c}=z_{T 0}+\lambda z_{\delta c}
\end{array}\right.
$$

II.4 Exporting: The meshes of both compensated tools are exported, the FEM simulation is run again and the deviations, after compensation, are calculated again.

The algorithm can be applied iteratively and the whole procedure is repeated until convergence, i.e. until the deviations between the simulated and designed parts are within the tolerance interval chosen by the designer.

\section{Numerical Results}

Before applying the compensation routine, the accountability of the FEM model must be verified. With respect to failure limits, experiment no. 7 is very useful, where the maximum force is applied on the blankholder. In the real tests, fracture occurred at this high level of draw-in restraint. The experiment and the simulation show the same location of the blank fracture localized at the deepest corners of the component (Figure 8). No other simulation nor experimental case had occurrence of fracture. Both in simulations and in real tests, no macroscopic wrinkling was detected. The errors across the cross section A-A between the experimental and numerical profiles are included in the range $[-0.23$; $+0.24]$ for test no. 2 and $[-0.20 ;+0.17]$ for test no. 3 . These levels of geometrical accuracy are in the typical range of FEM models with shell elements; they are acceptable for general purposes but they seem to be too large for a reliable compensation routine. However, it is interesting to observe the comparison given in Figure 9, which is limited to test case no. 2 for brevity. In this figure, the error of the FEM profile with respect to the designed part is compared to the error (already given in Figure 2) between the actual CMM measured profile and the designed part.

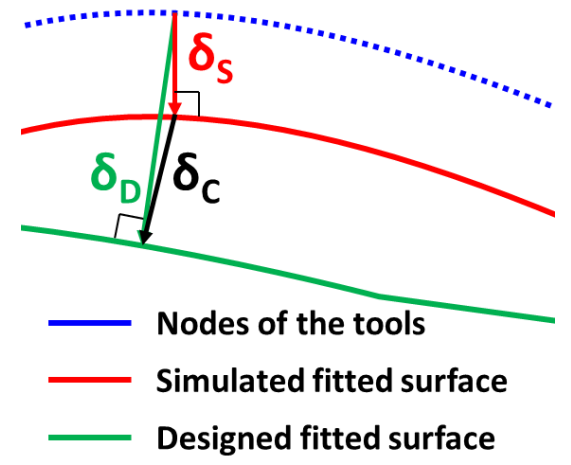

Figure 7: computation of the new tool nodes.

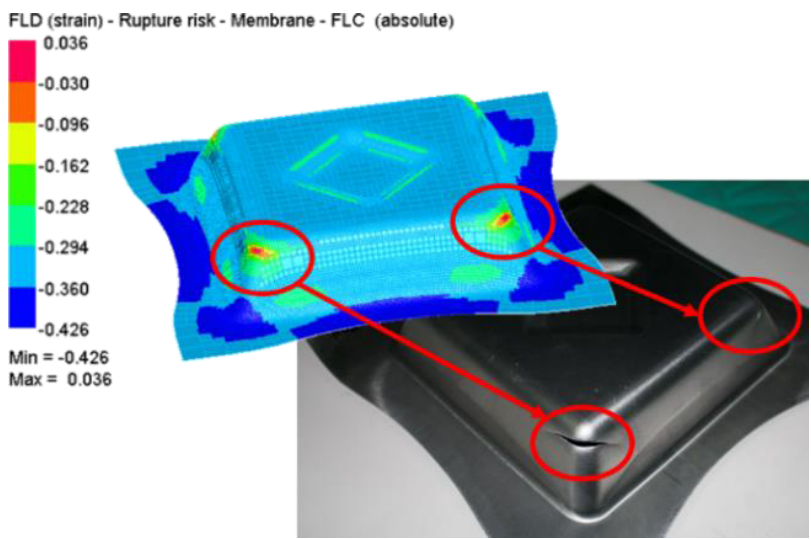

Figure 8: fracture localization - experiment no. 7. 


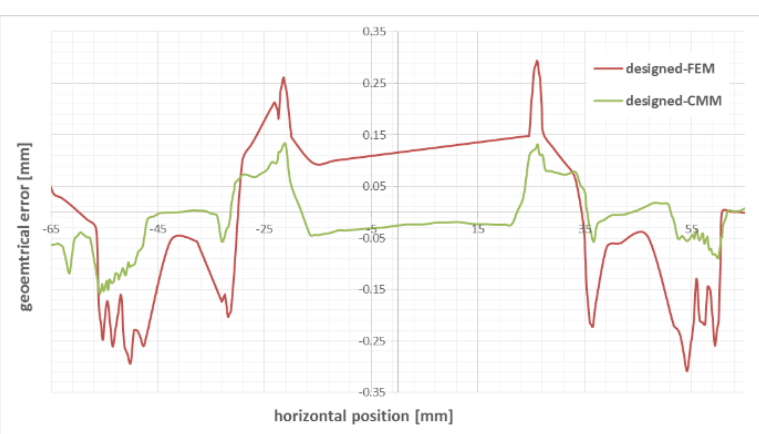

Figure 9: errors between FEM and experimental profile for experiment no. 2 .

Although the numbers are obviously different for the two profiles, (with a larger amplitude for the FEM error) the shape of the two profiles is surprisingly similar, with a clear indication of accountability of the FEM model.

In order to demonstrate the use of the proposed compensation technique, it has been applied to test cases no. 2 and no. 3 .

TEST CASE no. 2. This test case is run with $\mathrm{BHF}=9.8 \mathrm{kN}$. As the first simulation run, the tools are simply built as an offset of the final designed part middle surface. The plot of the deviations between this simulated part and designed components is shown in Figure 10, for the whole part, excluding the flange. The simulated component deviates considerably from its designed geometry in a range between $[-0.24 ; 0.44] \mathrm{mm}$ and only $78.53 \%$ of the calculated deviations lays within the range of $[-0.14 ; 0.15]$. The maximum positive or negative values of deviation are located on both the convex and concave corners. This corresponds to a large deformation of both tools on the corner radii.

The algorithm performed an automatic compensation of the tools geometry, with $\lambda=1$. As a result of the first compensation, the punch and die corners radii have been modified by the algorithm in order to reduce the deviations shown in Figure 10.

After the compensation, the range of the deviations between the designed and simulated components is reduced. In Figure 11, the results of the first iteration are shown. The range of the deviations, calculated in the normal direction, lays within the range $[-0.22 ; 0.15] \mathrm{mm}$.

This error range is not much different from the one in Figure 10 (obtained before compensation), but it must be noted that the $96.95 \%$ of the surface is within the range [$0.12 ; 0.10] \mathrm{mm}$. Furthermore, the increase of calculated effective stress on the tools is limited to only $1 \mathrm{MPa}$ on the punch and $7 \mathrm{MPa}$ on the die. This means the proposed algorithm is able not only to reduce the geometrical errors but also to save the impact on the expected tool life.

The time required for 1 simulation (run with 8 processors) and for 1 tool compensation (with 1 processor) is summarized in

Table 4.
The procedure described in Section 4 is iterative, it can be repeated with constant or variable values of the correction coefficient $\lambda$.

In Figure 12 the RMS value of the deviation vector is plotted vs. the number of iterations, with constant $\lambda$-value. The figure shows that the RMS values of the deviation vector decreases drastically after the first iteration and rapidly converges towards a stable solution. A very similar trend had been observed also in a previous work [15], and this indicates a typical behaviour of the proposed routine.

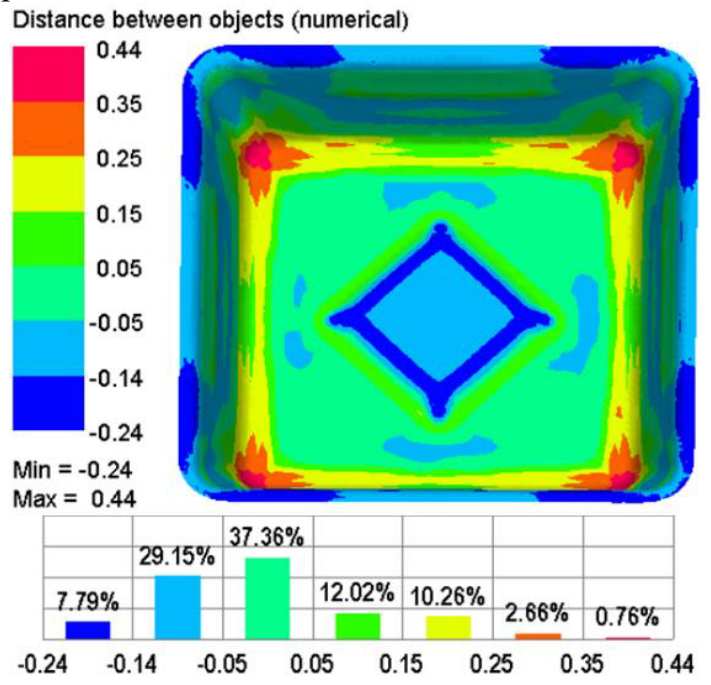

Figure 10: $\delta$-plot before compensation; isometric and top views, units in [mm].

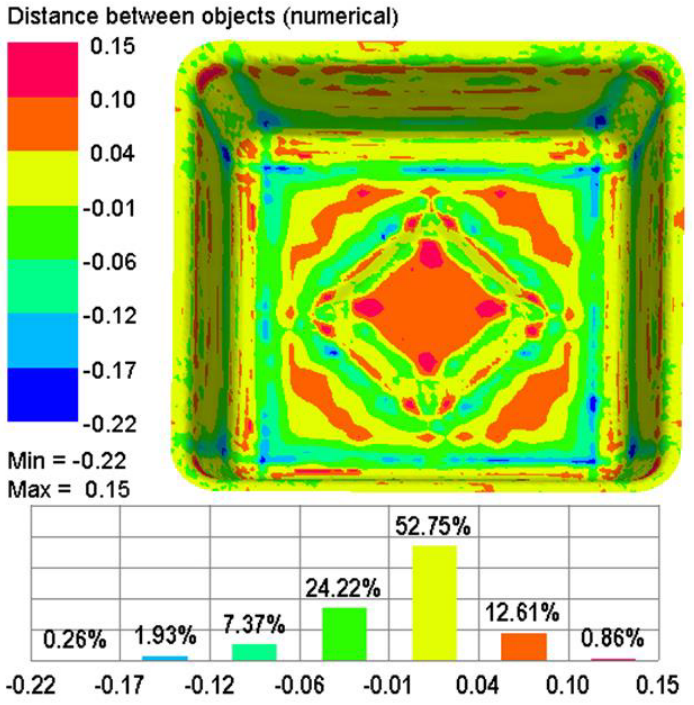

Figure 11: deviations between designed and optimized components after one iteration. 
Table 4: time needed for 1 iteration in compensation of tests case no. 1

\begin{tabular}{|c|c|}
\hline Operation & Time needed \\
\hline Simulation -3 stages & $4330 \mathrm{~s}$ \\
\hline Reading blanks mesh & $2 \mathrm{~s}$ \\
\hline Parameterization & $1 \mathrm{~s}$ \\
\hline Compensation & $60 \mathrm{~s}$ \\
\hline Save tool mesh & $0.1 \mathrm{~s}$ \\
\hline Total & $\mathbf{1 1 5 5 7} \mathbf{~ s ~ ( 1 . 2 3 ~ h ) ~}$ \\
\hline
\end{tabular}

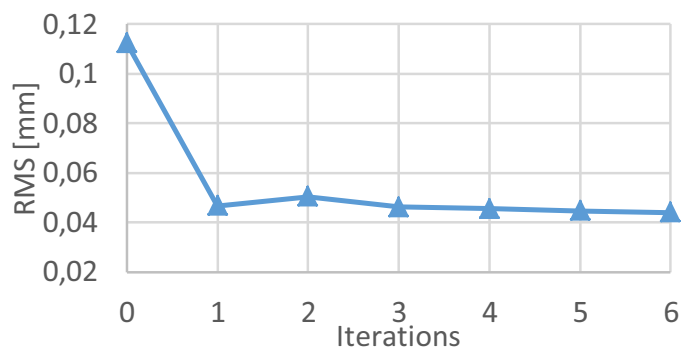

Figure 12: norm of deviation vector vs. iteration for test case no. 2

TEST CASE no. 3. This test case is run with $\mathrm{BHF}=14.7 \mathrm{kN}$. Again, in the first simulation run, the tools are built as an offset of the final designed part middle surface.

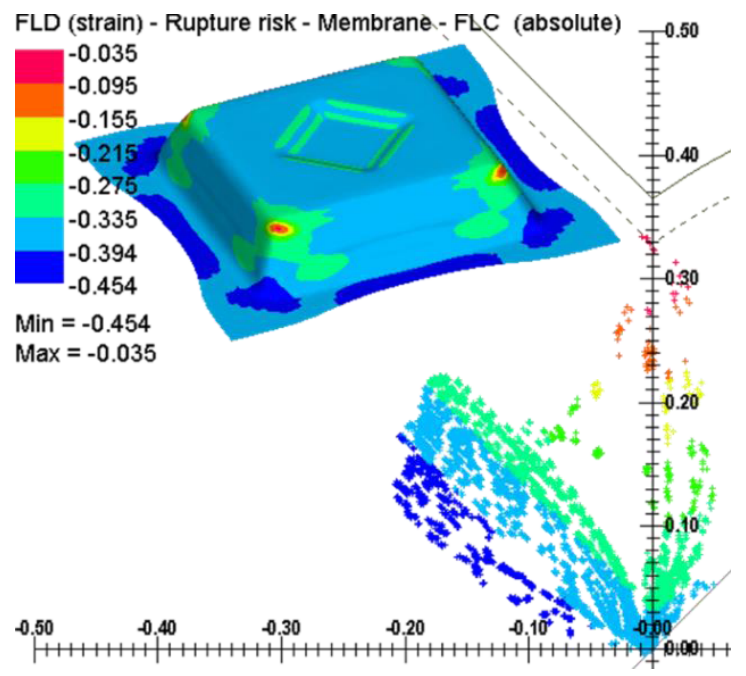

(a)

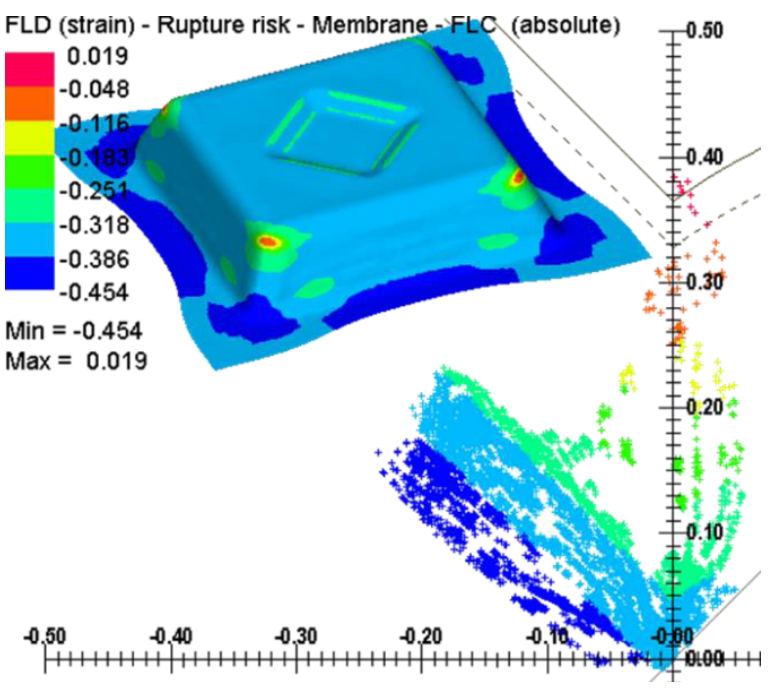

(b)

Figure 13: FLD map of test case no.3 before compensation (a) and after compensation (b).

The strain map, expressed in terms of distance from the FLD line, is shown in Fig. 13a. After the tool geometry compensation, the state of strain on the blank obviously changes, and the resulting FLD plot is shown in Fig. 13b, where a large risk of fracture is evident. In other words, in order to reduce the geometrical errors, the routine tends to increase the level of strain. This behaviour is typical since it has been observed also for test case no. 4 and for other tested geometries. As a result, the procedure described in Section 4 can be framed in a more general algorithm, which involves the selection of the correct level of BHF and the evaluation of the risk of fracture. This general algorithm is summarised in Fig. 14.

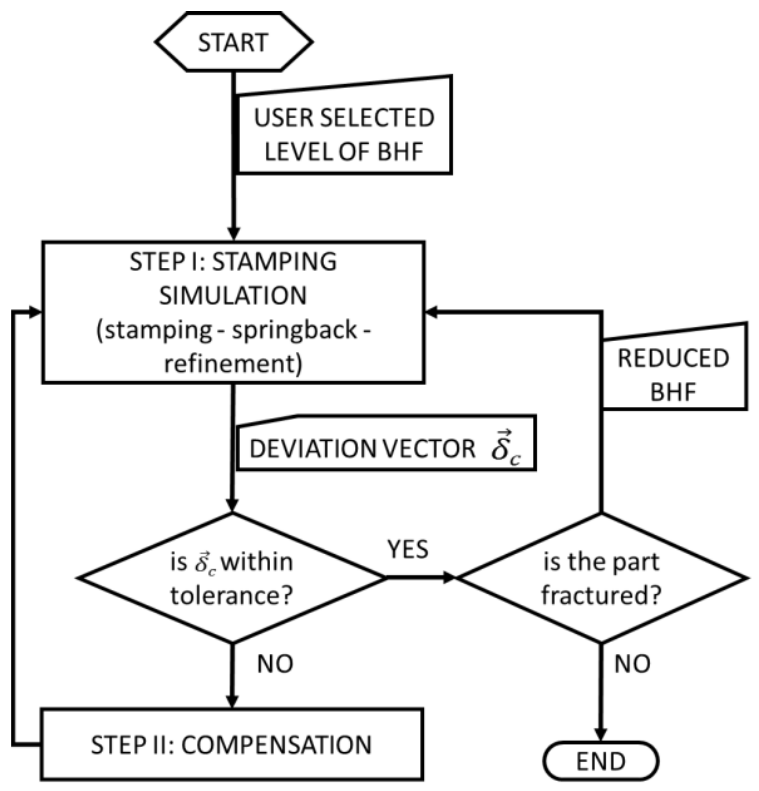

Figure 14: General compensation algorithm 


\section{Conclusions}

In this paper, a compensation algorithm has been proposed, based on the displacement adjustment (DA) approach. The proposed method allows to evaluate and reduce the deviations between the simulated component and the designed one.

The algorithm, based on the reduction the normal deviation vector, can be applied iteratively. It generates an increase of the strain level in the final workpiece, as a consequence of the change in the shape of the tools. For this reason, the an iterative algorithm has been proposed, which takes the risk of fracture into account. When the results of the compensation algorithm are far from the risk of fracture, the algorithm rapidly yields a satisfactory solution already after the first iteration.

\section{Acknowledgments}

This project has been partly funded by the Italian National Cluster project "Fabbrica intelligente", CTN01_00163. Mr. Alessandro Donghi is gratefully acknowledged for his precious help in the development of the experimental tests case.

\section{References}

1. Malhotra R, Cao J, Ren F, Kiridena V, Cedric Xia ZZ, Reddy NV. ASME. J. Manuf. Sci. Eng. 2011;133(6):061019-061019-10. doi:10.1115/1.4005179.

2. Son C-Y, Jeon Y-P, Kim Y-T, Kang C-G., Journal of Engineering Manufacture. 2012 Jan 30;226(5):909918.

3. Ramezani M, Ripin ZM, Ahmad R. CIRP Journal of Manufacturing Science and Technology. 2010 Jan;3(3):196-203.

4. Ramezani M, Ripin ZM. The International Journal of Advanced Manufacturing Technology. 2011; 59(58):491-505.

5. Strano M.: Journal of Engineering Manufacture. 2006; 220(8):1305-1315.

6. Vollertsen F, Breede R, Beckman M. Proceedings of the Institution of Mechanical Engineers, Part B: Journal of Engineering Manufacture. 2001 Jan 1;215(7):977-990.

7. Cai Z-Y, Wang S-H, Xu X-D, Li M-Z. Journal of Materials Processing Technology. 2009;209(1):396407.

8. Chua CheeKai, Leong KahFai, Liu Zhong Hong, Handbook of Manufacturing Engineering and Technology, Spinger, 2014, p. 1-22.

9. Park, Y., Colton, J.S., 2005. J. Manuf. Sci. Eng. 127, 116.

10. Longhi B. H., Eid C., Brynmorgen Press, 2013.
11. YANG, Xiang An; RUAN, Feng. International Journal of Mechanical Sciences, 2011, 53.5: 399406.

12. SHEN, Guozhe, et al. In: AIP Conference Proceedings. IOP INSTITUTE OF PHYSICS PUBLISHING LTD, 2005. p. 334.

13. W.H. Press, S.A. Teukolsky, W.T. Vetterling, B.P. Flannery, Numerical Recipes in C, Cambridge University Press, 1992.

14. Karafillis A.P., Boyce M.C., International Journal of Machine Tools \& Manufacture 1996; 36 (4): 503-36.

15. Iorio, L., Strano, M., \& Monno, M. (2015). In Proceedings of the ASME MSEC International Conference, Vol. 1: Processing (p. V001T02A089). Charlotte, NC (USA).

16. M. Amarandei, Antonio Virga, K-N. Berdich. Materiale Plastice, 2013; 50: 84-87.

17. SINTEF, Geometry/GoTools, https://github.com/SINTEF-Geometry/GoTools.

18. Lee, S., Wolberg, G., \& Shin, S. Y. (1997). Visualization and Computer Graphics, IEEE Transactions on, 3(3), 228-244. 